\title{
Escritura en la formación docente: los géneros de la práctica
}

\author{
Writing in the teachers formation: the genres of the practice
}

\author{
Gustavo Bombini* \\ Paula Labeur ${ }^{2 * *}$
}

Recibido: 8 de enero de 2013 / Aceptado: 18 de abril de 2013

\section{Resumen}

La relación entre práctica docente y escritura se constituye en un tipo de relación hasta ahora escasamente indagada y que viene demostrando cierta productividad por su impacto directo en los procesos de enseñanza y formación. El propósito de este artículo es dar a conocer los resultados de los investigaciones relacionadas con experiencias de formación docente en el área de letras que dan relevancia a la práctica de escritura como configuradora de los procesos de construcción y de reflexión de la práctica de la enseñanza.

Palabras clave: formación docente, prácticas de enseñanza, prácticas de escritura, lengua y literatura.

Abstract

The relationship between teacher trainings and writing is a highly productive, albeit scarcely investigated one, until now. It has been showing an interesting amount of productivity because of its direct impact on both the teaching and training processes. The purpose of this paper is to show some threads in the inquiry related to our experiences in the training of teachers within the Language Arts area, that show the relevance of a writing practice as a weft for the processes of building and reflecting about teaching.

Key words: teachers' training, teachers' training practice, writing practice and language Arts \& Literature.

En este artículo se presentan los resultados de investigación producidos en el ámbito de la Cátedra Didáctica Especial y Prácticas de la Enseñanza del Profesorado de Letras de la Universidad de Buenos Aires (Argentina), espacio en el que se realizan las prácticas o las residencias docentes, es decir, el desa- rrollo de las primeras clases a cargo de los alumnos que en breve ejercerán como profesores en el sistema de la enseñanza secundaria y en otros niveles. En el desarrollo de esas primeras experiencias se producen textos que cumplen la función de planificar la práctica por realizar, así como también otros que

1 * Licenciado y Doctor en Letras de la Universidad de Buenos Aires. Profesor e investigador de la Universidad de Buenos Aires (UBA), Universidad Nacional de San Martín (UNSM) y Universidad de la Plata (UNLP). Miembro del equipo de la Cátedra de Didáctica Especial y Prácticas de la Enseñanza de la Carrera de letras de la Facultad de Filosofía y letras de la UBA. Especialista en formación docente y escritura, didáctica de la lengua y la literatura, políticas de lectura y literatura infantil. Correo electrónico: gbombini@gmail.com.

2 **icenciada en Letras de la Universidad de Buenos Aires (UBA). Profesora adjunta de la Cátedra de Didáctica especial de la UBA. Coordina la Licenciatura en enseñanza de la lengua y la literatura de la UNSAM y se desempeña como docente en el Instituto Alicia Moreau. Es Miembro del equipo de la Cátedra de Didáctica Especial y Prácticas de la Enseñanza de la Carrera de Letras de la Facultad de Filosofía y letras de la UBA. Correo electrónico: perdidosentokio@gmail.com. 
acompañan las tareas de registro y el análisis de las prácticas ya realizadas, y que son el soporte de los procesos de reflexión acerca de la práctica.

En este marco se sostiene que existe una relación productiva entre los procesos de escritura, en cuanto los tipos de texto que se escriben acerca de la práctica y los modos en que se configura la propia práctica y la reflexión sobre ella. Precisamente, esta investigación se ha propuesto indagar sobre géneros posibles, entre los históricos y habituales, y los géneros nuevos productos de la práctica.

Se sabe que la formación de docentes para todos los niveles del sistema educativo plantea permanentes desafíos referidos a los modos en que se produce el complejo proceso - con implicaciones en lo subjetivo, lo social y lo institucional- por el que un alumno deja esa condición y rápidamente se convierte en docente. Este proceso involucra aspectos específicamente disciplinarios, pues el docente, maestro o profesor enseñará lengua, matemática, ciencias sociales o ética, y asimismo involucra aspectos específicamente prácticos que hacen a la construcción del objeto de enseñanza - acaso como reformulación del objeto de conocimiento disciplinario- y que habitualmente forman parte de la producción los campos de las llamadas didácticas generales y didácticas específicas. Es en la trayectoria de esos campos disciplinarios que se reconocen y se sistematizan los modos en los que históricamente se han producido los procesos de enseñanza y es desde donde se ha prescripto o normativizado acerca del hacer en el aula.

Esta perspectiva prescriptiva o normalizadora ha sido puesta en cuestionamiento por nuevas miradas que han buscado repensar las tradicionales concepciones acerca de la didáctica y de sus relaciones con la práctica misma. Ha sido el reconocimiento de la práctica de la enseñanza como práctica social lo que ha jerarquizado una nueva agenda de la didáctica que sale en busca de un conocimiento nuevo acerca de las prácticas de enseñanza (Edelstein y Coria,
1996). Podríamos decir que históricamente las didácticas han venido produciendo conocimiento más prescriptivo que descriptivo y en este sentido es que sabemos poco acerca de las prácticas efectivas del aula, seguramente no acerca de las deseadas o recomendables, sino acerca de las que efectivamente ocurren. Las orientaciones socioantropológicas en el campo de la didáctica propician una suerte de giro copernicano en relación con la idea de práctica y el modo en que la disciplina las aborda; y, en este sentido, son los aportes conceptuales y metodológicos de los estudios de las narrativas en educación (Michael Connelly y Jean Clandinin, 1994; Cochran-Smith y Lytle, 2002) de los enfoques biográfico-narrativos (Bolívar et al., 2001) y de la etnografía escolar (Bertely-Busquets, 2000) los que han dado relieve y precisión a estos desarrollos y que conforman la base del trabajo de escritura de las prácticas que pretendemos llevar adelante. A los marcos referenciales antes mencionados se les sumarían los aportes de los estudios didácticos acerca de la escritura y sus desarrollos en los ámbitos de los denominados talleres de escritura (Pampillo, Aren, Klein, Méndez y Vernino, 2009).

Es en el proceso de la formación docente y, más específicamente, en el momento en que el estudiante, futuro profesor, dicta sus primeras clases es cuando se ponen en tensión la fuerza de la intencionalidad explícita de la propuesta por desarrollar con los avatares que se producen en la escena de la enseñanza. Los profesores a cargo de este trayecto, habitualmente denominado prácticas docentes o residencia docente, despliegan un sinfín de dispositivos y estrategias para garantizar que este escenario de tensiones encuentre una resolución interesante que evite la exigencia y la rigidez del cumplimiento de lo planificado, y que no niegue y recupere de manera productiva imprevistos, reencauzamientos, reformulaciones, devenidos del desarrollo de la propia práctica (Robin, 1996).

Dentro de la tradición de la realización y guía de las prácticas, uno de los requerimientos propios del 
momento de dictado de las clases de iniciación a la docencia es la planificación que anticipa las tareas por desarrollar en el aula. En general, y como producto de las tendencias didácticas de corte tecnocrático más o menos dominantes desde la década del sesenta, lo requerido es la producción de una grilla en la que se detallan, organizados a partir de columnas, los campos que permitirán comprender y explicar los sentidos y las orientaciones de la tarea por desarrollar. De este modo, se irán presentando los ítems de las distintas columnas de la planificación, a saber, objetivos, contenidos, actividades, tiempo y observaciones. El producto final será seguramente el resultado de un proceso de trabajo en sucesivas versiones, con numerosas correcciones y vuelta atrás, hasta llegar a la versión definitiva.

En esta resolución y acompañamiento de las tensiones que se producen en la práctica de enseñanza, los dispositivos de escritura que se propongan cumplirán una función significativa o configuradora de la propia práctica. Se podrá afirmar y se verá en el desarrollo de este artículo- que existe una relación solidaria y productiva entre aquello que se escribe acerca de la práctica (de manera anticipada, durante su desarrollo, a posteriori de ella) y el modo en que esa práctica se va desarrollando. En este sentido, las investigaciones de corte cognitivo acerca de la escritura han hecho hincapié en el valor epistémico de la práctica de escritura; tal como lo afirman Alvarado y Cortés (2001): “todos esos abordajes coinciden en que la escritura promueve procesos de objetivación y distanciamiento respecto del propio discurso [...] ese descentramiento permite la revisión crítica de las propias ideas y su transformación”. Transpolada esta idea al campo de las prácticas docentes, podríamos decir que en cada situación de intervención docente se produce un proceso de producción de conocimiento didáctico en términos de microdecisiones que se ponen en juego en el trabajo del docente en el aula, las que habrán de ratificar o rectificar aquellas otras decisiones iniciales, que son las que se inclu- yeron en la planificación. De esas microdecisiones tomadas antes, durante y después de la práctica, se nutre un complejo dispositivo de escritura que va acompañando y sosteniendo el desarrollo y la reflexión acerca de la práctica y moldeando el proceso de formación del futuro profesor.

\section{En busca de nuevos géneros}

El desafío de cambiar el paradigma de trabajo en la didáctica y consecuentemente en el trabajo con la formación y con la enseñanza propicia el desarrollo de líneas de investigación centradas en la indagación práctica y teórica de nuevos y variados dispositivos de escritura que complejicen el proceso de construcción y reflexión sobre las prácticas, y que permitan observar la productividad de la escritura durante estos procesos. Esta complejización de las consideraciones acerca de la escritura de la práctica conlleva un necesario replanteo de los géneros posibles para esa escritura en términos de revisión de tradiciones y de nuevas invenciones para satisfacer las necesidades de esta perspectiva de trabajo. Por un lado, sabemos que existe una tradición interesante en relación con la escritura de los docentes que se remonta a los momentos fundacionales de los sistemas educativos y que tiene su manifestación en diarios de maestros, en cartas, en artículos firmados en revistas educativas, así como en distintos formatos, ligados a las exigencias burocráticas, como carpetas de actividades, cuadernos de actuación y otros géneros anteriores o coexistentes con la planificación a manera de grilla. Muchas experiencias docentes de cierto corte innovador, muchas referencias sobre el impacto en las aulas concretas de discusiones didácticas y pedagógicas son reconocibles hoy, gracias a esas escrituras. En este sentido, la escritura propicia la existencia de un saber acerca de la práctica sobre el que es posible volver desde la investigación histórica y etnográfica acerca de la práctica.

Replantear hoy el tema de los géneros de escritura de la práctica en el contexto de la formación, 
invita a una producción inventiva, que de algún modo recupere algo de esa historia, pero que a la vez responda a ciertas condiciones pragmáticas propias de las escenas de formación, como lo son las primeras clases que desarrollan los practicantes. Asimismo, la cuestión de la escritura como asunto transversal afecta todos los procesos de formación y no solo los que se dan en el contexto de las prácticas docentes. La cuestión de la escolarización académica y el manejo fluido de ciertos géneros asociados al estudio, como lo son las evaluaciones escritas o las monografías, vienen siendo asunto de preocupación de muchos especialistas y de las propias políticas educativas. La propuesta de escritura de las prácticas parece cuestionar los límites genéricos, a la vez que propicia un modo de construir conocimiento, una posición epistemológica diferente por cuanto lo que se propone es escritura sobre y a partir de las prácticas; un conocimiento empírico y experiencial pocas veces legitimado en el campo de la producción académica.

El mejor modo de exponer estos avances de investigación será ir presentando los géneros propuestos, considerando sus condiciones pragmáticas de uso y su paulatina aparición en la escena de las prácticas de modo que permita comprender sus sentidos en el propio contexto de su producción (Bombini et al., 2012).

\section{Guion conjetural}

En la producción de la situación de prácticas se hace necesario, en primer lugar, el trabajo con textos de anticipación de las prácticas por realizar. En el marco de esta investigación, se viene ensayando el trabajo con el género bautizado guion conjetural.

Cuando llega el momento de llevar a cabo la práctica docente de residencia, cada alumno escribe un guion conjetural (Bombini, 2002, 2004). En él imagina cómo serán sus clases, cómo desarrollará los temas que haya elegido o le hayan asignado, qué hará, qué dirá, qué propondrá hacer a sus alumnos e imagina también qué harán sus futuros alumnos en sus clases, cómo espera que reaccionen a sus propuestas. El guion conjetural es al mismo tiempo un ejercicio de imaginación y de toma de decisiones en el que los saberes disciplinares son pensados desde el lugar de la enseñanza y en contexto; su autor hipotetiza en un aula concreta con sujetos concretos, entre los que está él mismo, y, desafiando el lugar común de la neutralidad del saber escolar, el guión se escribe en primera persona. Las situaciones y contenidos son presentados por un "yo" que escribe lo que imagina para su futuro en el aula en un texto narrativo que tiene una doble recepción: el mismo autor desdoblado en una situación futura, y su tutor y compañeros de cursada a los que cuenta cómo será su tarea en el aula durante las horas de la práctica docente.

En la primera clase, planeo aclarar que durante un mes estaremos viendo literatura de terror. Pienso que a los alumnos les gustará mucho esta elección ya que imagino que es un género muy valorado por los adolescentes. Seguramente me preguntarán qué textos vamos a ver y si vamos a ver una película del género. Si esto sucede, les puedo aclarar que tanto leeremos cuentos como veremos videos y pinturas que puedan estar relacionados con el género.

Les diré que creen grupos de no más de 4 personas y repartiré distintos cuentos pertenecientes al género terror. Pediré que cada grupo designe a un lector (que se encargará de leer el cuento en voz alta para que el resto del grupo escuche), a alguien que tome nota acerca de las características que el grupo considera son propias del género, y los otros dos alumnos serán los expositores.

Cuando todos los grupos finalicen las lecturas los alumnos expositores comentarán al resto de la clase acerca del cuento que han leído. Una vez finalizado el intercambio de "experiencias", cada grupo leerá en voz alta aquellas características anotadas acerca del género. Se hará una puesta en común e iré anotando en el pizarrón todo lo que los alumnos digan. 
Busco la reflexión grupal acerca de lo que consideramos "terror". Y trataremos de llegar a una conclusión. Les pediré que anoten en sus cuadernos/ carpetas lo que pudimos sacar como conclusión, aclarándoles que de ahora en más revisaremos (completaremos, modificaremos) la definición a la que llegamos. Pienso que dirán que en la literatura de terror hay presencia de personajes extraños, "monstruos", fantasmas, lugares oscuros y sombríos, y muertes. Por lo tanto, intentaré en las clases siguientes problematizar esa definición, proponiendo textos que "entren en crisis" con esa definición. (C.J-J.P.B, 2013)

Como un mapa posible de acción durante la práctica docente, el guion es una especie de diario de viajero, el espacio textual que permite - mientras se está escribiendo- reflexionar sobre la práctica, pensar acerca de ella y pensarse en ella. De la mano de estas reflexiones, el componente explicativo-argumentativo entra en el guion: cada decisión tomada por el escritor debe ser expuesta de modo que convenza de su pertenencia a un marco teórico y su conveniencia en el contexto en el que ocurrirán las clases tanto a quien lo escribe como a quienes lo acompañan en este proceso.

En esta clase sería interesante, pero no sé si me voy a animar, tratar de ver algo de la "ironía" de Ovidio; en muchos aspectos, yo pienso que hay fuertes elementos paródicos en Metamorfosis, y algunos que llevan la idea de la transformación casi hasta el absurdo, como por ejemplo cuando Apolo le da besitos al árbol. También, me gustaría ver, retomando el concepto de intertextualidad, cómo fue tratado el mito posteriormente en la literatura y el arte, qué significación se le dio y cómo fue leído. Creo que acá voy a tener que hacer una búsqueda de reelaboraciones, porque las que encontré hasta ahora, en Garcilaso, Quevedo y Bernini me aburren un poco, y me gustaría ver algo menos clásico (se aceptan sugerencias). (G.C.F, 2013).

Desde una perspectiva etnográfica, la narración aparece en el guion como el género de escritura para dar cuenta de lo que sucederá en el aula, de maneras de argumentar y de construir las prácticas de modo más amplio que la planificación tradicional, ya que el practicante tiene que imaginar qué pasará en el tiempo real en el que dará clases. No se trata de abstraer objetivos, contenidos, actividades, sino, por el contrario, de detenerse en el detalle de todo lo que se imagina que ocurrirá en esa clase que todavía no ocurre.

Los guiones conjeturales se discuten en reuniones semanales entre el practicante y su tutor con la participación del resto del curso, pero también entre reunión y reunión- por los foros que provee el aula de la plataforma virtual: cada alumno envía su guion al foro, y su tutor y sus compañeros lo comentan. La publicación en el aula virtual y la circulación de los guiones en el foro a la vista de todos los participantes constituyen, en el espacio académico, una nueva mirada sobre los textos escritos. Estos no resultan la producción personal de un alumno, sino que se enmarcan en un proyecto de escritura compartida: los primeros textos van mutando con la participación del tutor y los compañeros de curso. Estos guiones discutidos y retocados son, al mismo tiempo, insumos para la producción de los guiones todavía no escritos cuyos autores advierten, en los textos de los compañeros, una serie de sugerencias, propuestas y modificaciones que incorporarán cuando produzcan los suyos.

/Primer texto del alumno/. Empezaré la clase planteando un diálogo/debate que recupere algunas nociones sobre qué entienden los alumnos por género fantástico en sentido amplio, diciéndoles que deben recurrir no solamente a saberes escolares, sino también a la noción de fantasía planteada en la cultura popular, que está más al alcance de la mano, de modo que no sientan que deberían tener necesariamente una respuesta erudita.

/Primera intervención del tutor/ ¿Por qué partirías de un intento de definición y no de textos posibles para irse acercando a la definición desde los textos? Si propusieras el trabajo con leyendas urbanas 
¿Contarlas, discutirlas, ver cómo son y cómo circulan?

/Reformulación del alumno/ Bien, dejemos este brainstorming para cuando los chicos estén más adentrados en el tema, a ver si de ahí podemos ir definiendo y problematizando la definición de un género. Tomemos por caso la leyenda de la dama de blanco. Les propongo una actividad de lectura, previa introducción en el tema de las leyendas urbanas y luego, que con el relato base armen otro tomando solamente aquello que la leyenda tiene anclado en la realidad (o en lo similar a la realidad), para ver pautas de identificación de la poética de lo fantástico en rasgos muy generales. Después les voy a pedir que lo lean en voz alta unos cinco, seis alumnos al azar para generar un debate (en caso de que no todos coincidan) acerca de esa categoría de "lo real".

/Segunda reformulación del alumno/ Además, pensar, a partir de esa identificación y por oposición, los hechos sobrenaturales, e imaginar otros, proponiendo una lista de entre 6 y 8 hechos que puedan ser utilizados más adelante en los talleres de escritura que les voy a proponer. (F.B., 2013)

Los primeros comentarios en la mayor parte de los casos reorientan el enfoque de los primeros guiones: no resulta fácil encuadrar la práctica en un nuevo marco teórico que pretende desandar aspectos nodales de la práctica más tradicional, larga y arduamente aprendida como alumnos. Imaginar un docente que coordine lecturas y escrituras, que sitúe a los participantes de la clase en un lugar protagónico y les dé el espacio necesario para apropiarse del saber resulta un giro copernicano que requiere más de una revisión de los guiones, al tiempo que se pone a jugar la bibliografía específica frente a los problemas concretos que abre cada guion. Pronto, la participación en los foros fluye. Aparecen nuevas propuestas de actividades, textos que complementan los existentes, ideas para avanzar sobre las primeras aproximaciones.

Los practicantes escriben y rescriben los guiones pensando en nuevos modos de enseñar, en cómo los sujetos se apropian del saber. La perspectiva didác- tica que sostiene el pedido de reformulación de los guiones opera no solo en estas otras maneras de pensar la enseñanza, sino también en el modo de pensar los textos escritos y la escritura en sí misma. Como en un juego de cajas chinas, mientras los alumnos van reformulando su mirada sobre la enseñanza, reformulan sus expectativas respecto de los textos escritos que están produciendo. A diferencia del modo de circulación de la mayoría de los textos de la formación, el guion conjetural no es un texto personal y definitivo sujeto a la evaluación del docente, por el contrario, es un texto inicial, borrador, provisorio, siempre sujeto a modificaciones. Es también un texto polifónico en el que su escritor, primero, cruza su voz con las de sus futuros alumnos y la bibliografía, y luego la entrelaza con las de su tutor y sus compañeros de cursada. El guion es, entonces, un texto polifónico que siempre se está escribiendo sin llegar a una versión definitiva. Aun cuando los guiones resultan aprobados y esperan transformarse en acciones concretas en la práctica, los sigue atravesando un matiz de provisorio, de mutante, de indefinido: "resolveremos en el aula", "iremos viendo mientras ocurra" son anotaciones frecuentes del tutor en los márgenes de los escritos, advertencias de lo imprevisible de esa práctica social que es la enseñanza que no termina dejándose atrapar por la escritura.

\section{Autorregistro}

Con el guion aprobado, cada practicante comienza su práctica docente de residencia de la cual da cuenta con la escritura de autorregistros. Los autorregistros son narraciones que incluyen explicaciones y el desarrollo de argumentos en los que el practicante cuenta e interpreta qué pasó mientras estuvo dando clase. En el terreno de la práctica, con alumnos reales que hacen sus aportes y desvían en algunas situaciones el recorrido originalmente planeado, con los sucesos de la institución modificando el tiempo cronometrado mentalmente, el practicante actúa y toma nuevas decisiones. Porque el guion —conjetu- 
ral, ficcional, flexible, maleable - admite ser modificado según los devenires de lo que efectivamente ocurre en la clase; el autorregistro reflexiona sobre qué caminos marcados en el mapa se siguieron y dónde llevaron y de los golpes de timón que se fueron dando para acomodar ese texto mutante a los sucesos del aula.

A medida que las prácticas van transcurriendo, el guion conjetural y autorregistro se imbrican, el registro de lo que ocurre lleva a conjeturar posibles nuevos caminos por seguir. Hay que rehacer el mapa en función de emergentes insospechados y volver a imaginar los recorridos con un conocimiento del contexto que cada vez se hace más afinado y sutil.

Se anticipan a lo que el texto luego tratará y mencionan, por ejemplo, antes de que aparezca explícitamente en el texto, el aburrimiento que la escritura académica siempre escrita con el mismo molde produce. Tal vez podría haberme detenido en por qué anticiparon eso, qué indicios les daba el texto para llevarlos a esa hipótesis, que luego confirmará y qué efecto tiene eso para el lector. No me di cuenta en el momento. Marcan más adelante, como una característica del ensayo la libertad temática y formal. Nos deteneos entonces un ratito a discutir de qué manera si un ensayo tiene libertad de tema y de forma puede, si puedo escribir cualquier cosa de cualquier manera, entonces puedo hablar de un género ensayo y ponerle rótulo. El ensayo de Larrosa se va poniendo cada vez más interesante, los vi interesados en la lectura, pero es un ensayo largo y si lo leemos todo no vamos a tener tiempo para empezar a comentar los ensayos de los compañeros, así que corto la lectura a medio camino y les propongo que lo terminen de leer, les digo que realmente no tiene desperdicio y como los veo entusiasmados espero que lo terminen de leer para la clase que viene (R.V, 2013)

La escritura de guiones conjeturales y autorregistros - en la objetivación y el distanciamiento que promueve- invita a los alumnos a descubrir nuevas asociaciones, recrear nuevas situaciones y también al objetivar las escenas y conjeturas acerca de una clase, cuestionar representaciones y expectativas previas sobre lo que sucede en el aula, experimentar modelos de análisis, reflexionar acerca de las consecuencias de determinadas normas. Alterando el tradicional circuito cerrado que va del alumno al docente para volver al alumno, la escritura abierta y compartida - posibilitada por el soporte del aula virtual- llega al público amplio de una comisión de trabajo que está escribiendo sus propios textos y leyendo e interviniendo los de los compañeros de aventura, pero además ese canal admite una intervención casi diaria y en tiempo real de la práctica. En la mayor parte de los casos, los practicantes dan dos o tres clases por semana y los intercambios con su tutor y compañeros se producen inmediatamente, para posibilitar que estas intervenciones sean insumos para las prácticas posteriores. Casi diarias, abiertas, grupales, las escrituras vertiginosas que promueve el soporte virtual reconfiguran la situación retórica: la comunicación suele resultar menos jerárquica que en la clase presencial y se van tramando modos de confianza - dada por el intercambio personal casi cotidiano- que reconfiguran la comunicación virtual y presencial.

La escritura, como ejercicio sociocultural puesta al servicio de la práctica docente, muestra su poder epistemológico. En la tradición escolar y académica, el texto escrito suele cerrar un ciclo de aprendizajes hechos de otra manera. Lecturas, exposiciones, debates, charlas, discusiones sobre textos y problemas se llevan a un último momento en el que se escribe. Se escribe un parcial, se escribe un informe de lectura, se escribe una monografía. Son estos, textos definitivos y sujetos a la evaluación. Por el contrario, en los guiones conjeturales y en los autorregistros, la escritura es un insumo para pensar la práctica que se va construyendo, porque se escribe y en el mismo momento de escribir. Estos escritos son entonces textos provisorios y sujetos a las intervenciones del tutor y de los compañeros. No dejan de ser nunca borradores que pueden ( $\mathrm{y}$ son) modificados por es- 
tas participaciones y la misma puesta en práctica de los mapas de ruta en el contexto concreto y real del aula. El texto escrito, como herramienta para imaginar y pensar, para reflexionar y volver sobre lo conjeturado, para revisarlo y elaborar nuevas posibilidades, lo convierte no en el cierre de un aprendizaje, sino en un insumo para aprender acerca de la práctica de enseñanza. Tachado, coloreado con todas las posibilidades que ofrece el procesador de textos, con globitos de comentarios al margen, polifónico; el texto escrito no es nunca definitivo y puede modificarse todas las veces que sea necesario. Y el tachar, colorear, anotar, mezclar voces, al contrario de la mayoría de las situaciones que los alumnos recorren en su formación, no indican que el texto está mal, sino que creciendo y discutiendo, volviendo atrás para reflexionar, cambiar de rumbo el texto va dando cuenta del aprendizaje que se está produciendo porque se escribe.

/Texto 1 de alumno/ La clase comenzó a las 7:50 horas. Dedicamos la primera parte de la clase a la devolución de los trabajos que hicieron hasta ese momento.

/Tutor/ ¿Cuáles fueron los criterios de esa devolución?

/Texto agregado de alumno/ (Acá hago un paréntesis para responder a tu anterior devolución. La corrección de trabajos que realizamos constó sobre la base dos o tres criterios básicos: el primero tuvo que ver con el respeto de la consigna. Ese criterio atravesó los tres trabajos escritos. El segundo también fue ortografía y redacción. Lo explicito porque hicimos correcciones de ese tipo (al estilo marcar errores y cuestiones de redacción) pero no hicimos de esto un aspecto central de la corrección. El tercero tuvo que ver para los textos de Ciencia Ficción, la aparición de características discutidas en clase (como la anticipación de inventos, las tecnologías, la ciencia, el ideal de futuro) y para el trabajo de argumentación las cuestiones de la pretendida objetividad y la justificación, y la búsqueda de convencer, plasmadas en algunas estrategias que al momento de esta actividad eran más intuitivas).
/Texto 1 de alumno/ Al finalizar el reparto dijimos que cualquier duda que hubiera con las correcciones la podían consultar. Yo tuve una sola duda que se generó en relación al no cumplimiento de una de las pautas de la consigna en relación al texto de ciencia ficción. La consigna explicitaba que tenía que haber una tecnología que se saliera de control por lo que destiné unos minutos con el alumno a releer el texto y ver en dónde él había puesto ese elemento o cómo lo había hecho. Una vez que aclaramos que era un elemento faltante le aclaré que igualmente el texto a nivel de la trama y de otras características de la ciencia ficción que habíamos discutido estaban muy bien, pero que sólo había que ajustar o prestar atención a ese tema de la consigna.

/Agregado de alumno/ Tomando lo que vos me dijiste en la devolución de los registros anteriores, ya en la clase del martes tratamos de volver sobre los textos tanto al principio como al final. La verdad que fue productivo. La actividad de preguntarnos sobre qué estrategias habían usado generó mucho interés y nos permitió redondear cosas que tal vez habían quedado en el aire.

/Agregado de tutor/ ¿Quizás el interés viene de que están poniendo en foco los textos propios?

/Agregado de alumno/ Tal vez la expresión «en al aire» no fue la más acertada. La práctica con la escritura permitió que hubiera todo un soporte de experiencia (no en el aire sino en el saber de todos) que al momento de la discusión en clase y el dictado encontró una síntesis. Pero claro que eso hubiese sido una actividad vacía si no hubiésemos tenido la actividad de escritura como soporte. En relación a eso tengo que admitir que estoy bastante sorprendido. Tanto por la complejidad de las producciones de los chicos como por la conciencia que tenían de las estrategias sin siquiera haberlas tratado en profundidad «teórica». (A.A. 2013)

\section{Dos hojitas}

Una vez finalizadas las prácticas pedagógicas, los alumnos escriben un texto breve al que se le denomina dos hojitas. Dos hojitas es un fuera de género que no tiene más que tres condiciones: ocupar solo 
dos hojas A4 con los tipos e interlineados convencionales; dar cuenta e interpretar una escena de la práctica que por algún motivo resulte fulgurante al practicante y llevar un título. Si bien no está inscripta en la consigna, la primera persona suele recorrer las "dos hojitas" que se ven atravesadas por el asombro al descubrir en la práctica concreta todo aquello que se había discutido - con distancia y sospecha- con la bibliografía teórica. Algunos temas, en la acumulación de dos hojitas en el haber de la cátedra y con el paso de los años, se vuelven temas, el saber escolar transcurre por lógicas diferentes a las del saber disciplinar; los cruces son inesperados y bienvenidos; los sujetos que están en las aulas saben acerca de muchos temas; los procesos alquímicos de saberes se construyen en el aula entre estos sujetos y el docente y entre esos mismos sujetos; el modo expositivo de transmisión de conocimientos no resulta operativo en el contexto escolar; las lógicas de legitimación son extrañas a las académicas; debatir, escribir y comentar las lecturas parecen ser los modos más adecuados para lograr que ocurran las apropiaciones del saber disciplinar. Verse en el lugar docente reconfigura la visión acerca de la lectura: ya no se lee para sí, para aprender, sino para compartir, para hacer circular, para pensar en y con el otro que no es un par. La producción crítica o las lecturas oficiales toman otros rumbos cuando se trata, en el nuevo rol, de exponerse y contribuir al desarrollo de la lectura crítica poniendo a disposición las estrategias y saberes en juego, necesarios para abordar la literatura. Verse en el lugar docente reconfigura la visión acerca de la escritura: mucho más compleja que como habilidad instrumental para repetir saberes aprendidos, se constituye en una herramienta de exploración -por medio de la invención y la imaginación- de las posibilidades del lenguaje, de las reglas de la ficción; esta visión permite que los alumnos de los practicantes se posicionen como productores más que como repetidores en el mundo de la cultura.
Cada año, las dos hojitas de cada comisión de trabajo se organizan en un único documento que los practicantes reciben en sus correos electrónicos: encontrarse a sí mismos y encontrarse en la voz que los otros les muestra en pequeñas escenas -en las similitudes y diferencias - la complejidad de una práctica social, la de la docencia, que, al principio de la cursada de la materia, se mostraba como unívoca, sin fisuras, monótona, levemente aburrida, conocida por todos.

\section{Conclusiones}

Como cierre se puede concluir que el trabajo con la escritura viene demostrando su productividad en cuanto al reconocimiento y el abordaje de la complejidad de los procesos de enseñanza y de formación, lo cual propicia una perspectiva crítica tanto de los puntos de vista del alumno en formación como del propio formador que interviene activamente en proceso de la práctica, gracias al intercambio de lecturas y escrituras propiciado por estos textos.

Desde el punto de vista del alumno, la escritura en primera persona de distintos géneros de la práctica contribuye en términos de construcción identitaria en su proceso de aproximación paulatina a su nuevo rol profesional. Se trata de un posicionamiento que asume una voz que va dando cuenta de sus procesos de toma de decisiones, que muestra sus dudas y vacilaciones, explicita sus contradicciones y argumenta sus decisiones y sus modos de resolver los avatares de la práctica. Y, a la vez, vuelve - mientras lo comunica a su formador y a sus pares en formación- sobre su propio proceso de una manera crítica y transformadora.

Asimismo, y como fue anticipando, es destacable el hecho de que en los últimos años la incorporación de las nuevas tecnologías ha redundado positivamente en el desarrollo de esta perspectiva de escritura en las prácticas. Tutores y practicantes han ido escribiendo en una cadena de correos electrónicos 
articulada a partir de la función responder a todos, en grupos de correo en los que van y vienen textos que a la vez quedan disponibles, en un blog que permite colgar textos y hacerlos visibles para todos los alumnos y docentes de la cátedra, y actualmente de forma más institucionalizada en los distintos espacios que habilita el aula de la cátedra dentro del campus virtual de la facultad. La escritura en el contexto de la virtualidad propicia formas de producción y de circulación; lectura e intercambios inéditos a la hora de configurar grupos de prácticas que intercambian y reflexionan sobre sus experiencias a partir de textos. Además, falta explorar en las posibilidades que ofrece la multimodalidad a la hora de recurrir a otros lenguajes y expresiones estéticas que se vinculan con la cuestión de las prácticas de enseñanza y formación (películas, fotografías, ilustraciones, fuentes históricas, música, etc.).

Las líneas de investigación expuestas se han venido desarrollando en el ámbito de la Cátedra Didáctica Especial y Prácticas de la Enseñanza en Letras de la Facultad de Filosofía y Letras de la Universidad de Buenos Aires, en dos proyectos acreditados dentro del Programa UBACyT (2006-2009 y 20102012) - con antecedentes de puesta en práctica del guión conjetural realizados en Cátedra afín de la Universidad Nacional de La Plata entre 2000 y 2009. (Bombini, 2002) - que se han abocado al trabajo de escritura de géneros de la práctica, así como también de géneros ficcionales en relación con los procesos de formación en el campo de la didáctica de la lengua y la literatura y de la formación docente. Todas estas investigaciones se realizan a partir de materiales empíricos producidos en la misma instancia de formación de la cátedra. Cabe señalar en impacto de esta línea en cátedras afines, así como también en espacios de formación y en espacios docentes de otros niveles y modalidades (educación inicial, primaria, matemática, ciencias sociales, educación especial, educación superior, entre otros).

Finalmente, y en relación con el punto anterior, cabe mencionar que el tema central del proyecto postulado para el periodo 2013-2016 de la Programación Científica de la Universidad de Buenos Aires es el de la productividad de la multimodalidad en el proceso de formación docente.

\section{Reconocimientos}

En este artículo presenta los resultados de la investigación Cátedra Didáctica Especial y Prácticas de la Enseñanza del Profesorado de Letras de la Universidad de Buenos Aires., proyecto acreditado por el Programa UBACyT (2006-2009 y 2010-2012)

\section{Referencias}

Alvarado, M. y Cortés, M. (2001). La escritura en la Universidad. Repetir o transformar. Lulú Coquette. Revista de Didáctica de la Lengua y la Literatura, (1), 19-23.

Bertely-Busquets, M. (2000). Conociendo nuestras escuelas. Un acercamiento etnográfico a la cultura escolar. México: Paidós.

Bolívar, A., Domingo, J. y Fernández, M.(2001). La investigación biográfico-narrativa en educación. Madrid: La Muralla.

Bombini, G. (2002). Prácticas docentes y escritura: hipótesis y experiencias en torno a una relación productiva. Ponencia presentada en Primeras Jornadas de Prácticas y Residencias en la Formación Docente, Facultad de Filosofía y $\mathrm{Hu}$ manidades, Universidad Nacional de Córdoba, Córdoba, Argentina.

Bombini, G. (2004). Reinventar la enseñanza de la lengua y la literatura. Buenos Aires: Libros del Zorzal.

Connelly, M. y Clandinin, J. (1994). Relatos de experiencia e investigación narrativa. En J. Larrosa (Ed.). Déjame que te cuente, Ensayos sobre narrativa y educación (11-59). Barcelona: Laertes.

Cohran-Smith, M. y Lytle, S. (2002). Dentro/Fuera. Enseñantes que investigan. Madrid: Akal. 
Edelstein, G. y Coria, A. (1996). Imágenes e imaginación en la docencia. Buenos Aires: Kapelusz.

Pampillo, G., Aren F., Klein, I. Méndez, A., y Vernino, T. (2009). Escribir: antes yo no sabía que sabía. Buenos Aires. Prometeo.
Robin, R. (1996). Identidad, memoria y relato. La imposible narración de sí mismo. Buenos Aires: CBC-UBA. 